\title{
Y Aprendizajes Género y medios de comunicación: más allá del sexo
}

Sintesis:

En este estudio se examinó la relación de la identidad de género con la selección, interpretación y producción de consumos culturales televisivos en una muestra de jóvenes universitarios. La muestra incluyó 298 jóvenes de ambos sexos, de niveles socioeconómico bajo y medio de Bogotá. Para obtener la información se utilizó un cuestionario de auto-diligenciamiento. Los resultados mostraron que si existe relación entre los indicadores de la identidad de género examinados y la selección, interpretación y producción de consumos culturales

televisivos. El presente artículo únicamente

menciona los resultados que hacen referencia a las relaciones encontradas más allá del sexo de los participantes. El resto de los resultados serán objeto de un artículo posterior.

Palabras clave: género, sexualidad, identidad consumos televisivos.

Synthesis:

In this study was examined the relation between the genre identity and the selection, interpretation and production of cultural television consumptions in a group of young university students. The group included 298 young people of both sexes, low and medium socioeconomic levels from Bogotá. The information was gathered through a self taking survey. The results evidenced that it does exist a relation between the identity indicators of genre examined and the selection, interpretation and production of cultural television consumptions.

The present article only mentions the results that make reference to the relations found beyond the participants' sex. The rest of the results will be in the subject of a posterior article.

Key Words: Gender, sexuality, identity, consumption television
Ángela Rojas

Magíster en Género y Comunicación, Psicóloga. Docente investigadora de la Facultad de Ciencias de la Comunicación de Uniminuto.

e-mail: arrojas@uniminuto.edu

\section{La relación entre los medios de comunicación y el} género ha sido objeto de análisis y debate desde hace algunas décadas. Aunque en la actualidad se cuenta con numerosos artículos sobre el tema, no slempre se basan en datos investigativos.

Los estudios que han examinado el tema de género en los medios de comunicación han girado en torno a tres ejes principales: uno relacionado con el papel desigual que se otorga en los mensajes a los hombres y a las mujeres (Herold \& Foster, 1975; Luke, 1996; Ingham, 1997; Bonder, 1995: López, 2001; WACC, 2005; Flores y Sánchez, 2006); otro centrado en la influencia de los mensajes en la construcción de creencias, actitudes y comportamientos sexuales o de género en los públicos (Witt, 1996; Muramatsu, 2002; Charles, 2002, Vargas, Barrera, Burgos \& Daza, 2004; Bussey, K, y Bandura, A, 1999); por último, estudios o reflexiones sobre el género (aunque sería más correcto decir el sexo) como factor moderador del proceso de recepción (Orozco, 1996, 1998; Lozano, 2000, 2001; Martín Barbero, 2003)

En el caso del consumo mediático, gran parte del trabajo sobre género y comunicación, ha pretendido desentrañar el papel de los medios de comunicación en la construcción social y cultural de las identidades femeninas (Charles, 1996). En estos trabajos la mujer constituye, 
generalmente, el foco de atención debido a que se le incluye dentro de los grupos de población más vulnerables. Los resultados de estos trabajos han sido de gran utilidad para tomar conciencia de las inequidades encontradas y de esta forma sugerir cambios en los contenidos de los mensajes que se emiten y se ponen a disposición del público acerca de las mujeres. Sin embargo, una de las deficiencias actuales en lo estudios sobre género y comunicación es la omisión, prácticamente generalizada, del análisis de las identidades masculinas que se muestran, producen y refuerzan a través de la televisión. Omisión que sólo recientemente comienza a suplirse.

Cabe señalar que la mayoria de las investigaciones sobre los estudios de recepción y género han utillzado

w metodologías cualitativas, especiamente análisis etnográficos, entrevistas a profundidad, observaciones directas e historias de vida. En algunos casos análisis de contenido. Según la especialista en medios de comunicación Mercedes Charles (1996) las metodologias cuantitativas no gozaban de aceptación por parte de los investigadores de esta linea de estudio. Esta situación ha ocasionado la falta de disponibilidad de estudios comparativos entre reglones y paises, con perspectivas cuantitativas, que permitan sustentar la toma de decisiones políficas. Una consecuencia de esta circunstancia es la que plantea Orozco (2001), según la cual las pretensiones de transformación sobre la recepción mediática no se han dirigido a la formulación de grandes políticas públicas sino a propuestas más caseras para realizarse en microescenarios. Dado el impacto cultural que puede tener la comunicación mediática, la investigación que se sintetiza en este articulo pretende aportar información cuantitativa útil para la formulación de otras alternativas de transformación.

Adicionalmente, los grupos de investigación en los que se enmarca este estudio, consideran necesaria la combinación de las aproximaciones cuallitativas y cuantitativas para lograr una visión más amplia y completa de fenómenos tan complejos como los que aborda esta investigación: las identidades de género y los consumos culturales.

Otra de las grandes deficiencias de la mayoria de los estudios revisados sobre género es que, en general, el análisis se basa en establecer diferencias atribuibles al sexo $y$, realmente, no examinan el género como variable. Es decir, estos estudios asumen que si se observan diferencias entre hombres y mujeres estas pueden interpretarse como evidencias de diferencias de género'. En este trabajo se parte del supuesto de que aunque se dispone de información sobre las diferencias que existen entre las personas en función del sexo es poco lo que se sabe sobre la influencia de las diversas identidades de género, independientemente del sexo al que se pertenece.

Para los propósitos de este estudio, la identidad de género no se considera desde una sola perspectiva. de acuerdo con Egan y Perry (2001), se define como un constructo multidimensional que abarca al menos tres dimensiones: a) el sentimiento de compatibilidad psicológica con la propia categoría sexual, b) la presión social percibida para conformarse con los estereotipos

\footnotetext{
'El Sexo se refiere a las características biológicas que diferencian a hombres y a mujeres. El género hace referencia a los roles, responsabilidades y oportunidades asignados al hecho de ser hombre o ser mujer y a las relaciones socio-culturales entre mujeres y hombres y niñas y niños. Estos atributos, oportunidades y relaciones están socialmente construidos y se aprenden a través del proceso de socialización. El hecho de que una persona se identifique y esté satisfecha con su sexo, independientemente de su orientación sexual, no quiere decir que también se identifique y esté satisfecha con su genero, tal como la presente investigación lo ratificó.
} 


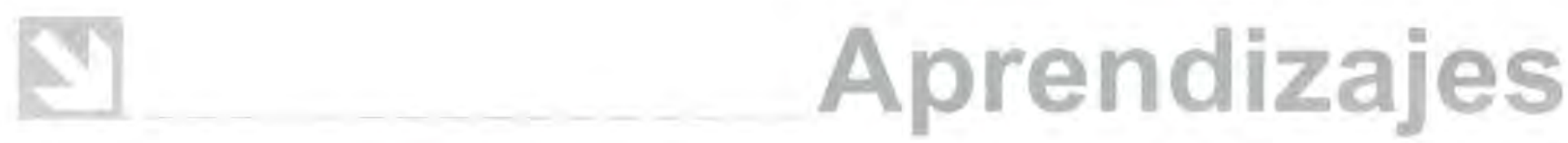

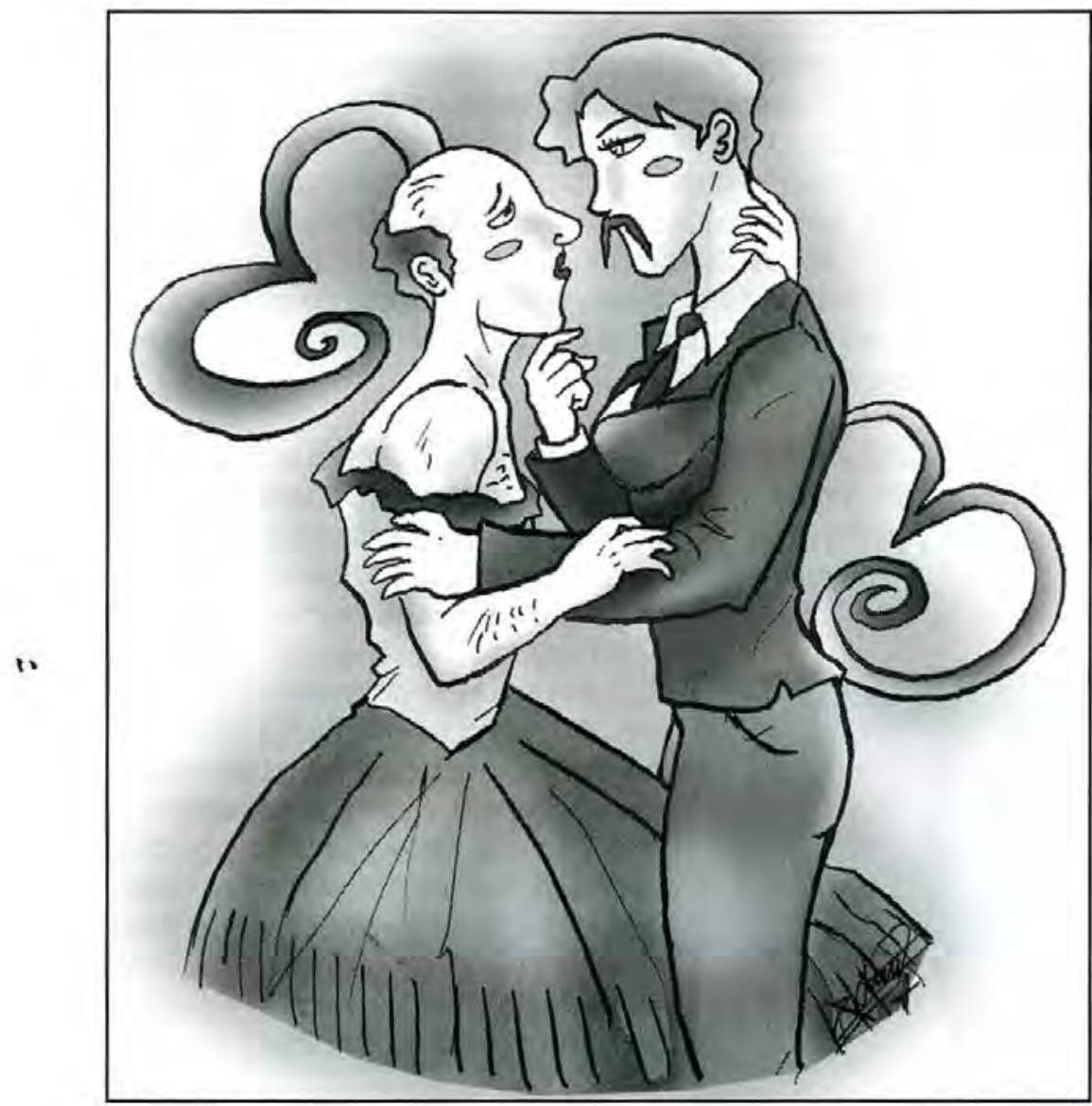

de género asignados al sexo biológico y c) las actitudes hacia los otros grupos como el sentimiento de que el sexo propio es superior. Estos tres aspectos se denominaron en su orden, para nuestra investigación: a) identidad con el sexo, b) identidad con el género y conformidad con los roles de género y c) conformidad con las actitudes sexistas.
La identidad con el sexo hace referencia a la adscripción de la persona a una de las dos categorias sexuales establecidas y la percepción de compatibilidad con el hecho de ser hombre o mujer. La identidad con el género corresponde a la percepción de compatibilidad general con la propia categoría de género, es decir, con el conjunto de normas y expectativas que socialmente regulan la forma de ser y de comportarse las personas en función de su sexo. Los roles de género representan el grado de conformidad con cada una de las normas y expectativas sociales examinadas acerca de cómo deben ser y comportarse las personas en función de su sexo. Las actitudes sexistas tienen que ver con la disposición de la persona a tratar de manera desigual a las personas por el hecho de ser biológicamente hombres o mujeres.

Dentro de nuestra línea de trabajo, se parte de considerar que la identidad de género implica valorar no sólo la identidad con el sexo, la identidad con el género sino también los roles de género y las actitudes sexistas. Estas cuatro variables son las variables rectoras del presente estudio.

\section{Género y consumo}

El consumo según la revisión hecha por Zukin y Smith (2004) se ha estudiado tanto en relación con cambios estructurales económicos y sociales, como en relación con cambios individuales en valores, actitudes y comportamientos. Los cambios individuales, dicen estos autores, desencadenan lo que se ha denominado consumo cultural. Desde la comunicación, el consumo 
cultural fue definido por García Canclini (1999) como el proceso de apropiación de los productos culturales por parte de los individuos. Los productos culturales son todos aquellos objetos materiales o inmateriales producidos por los seres humanos dentro de la cultura $y$, por lo tanto, poseen significados dentro de ella.

En los procesos de consumo cultural el valor simbólico prevalece sobre el valor de uso y de cambio, las personas consumen novelas, seriados, noticieros, "realities" o programas musicales, no por su utilidad práctica o por la posibilidad de intercambiarlos con otros productos, sino principalmente por el valor simbólico que representan.

r

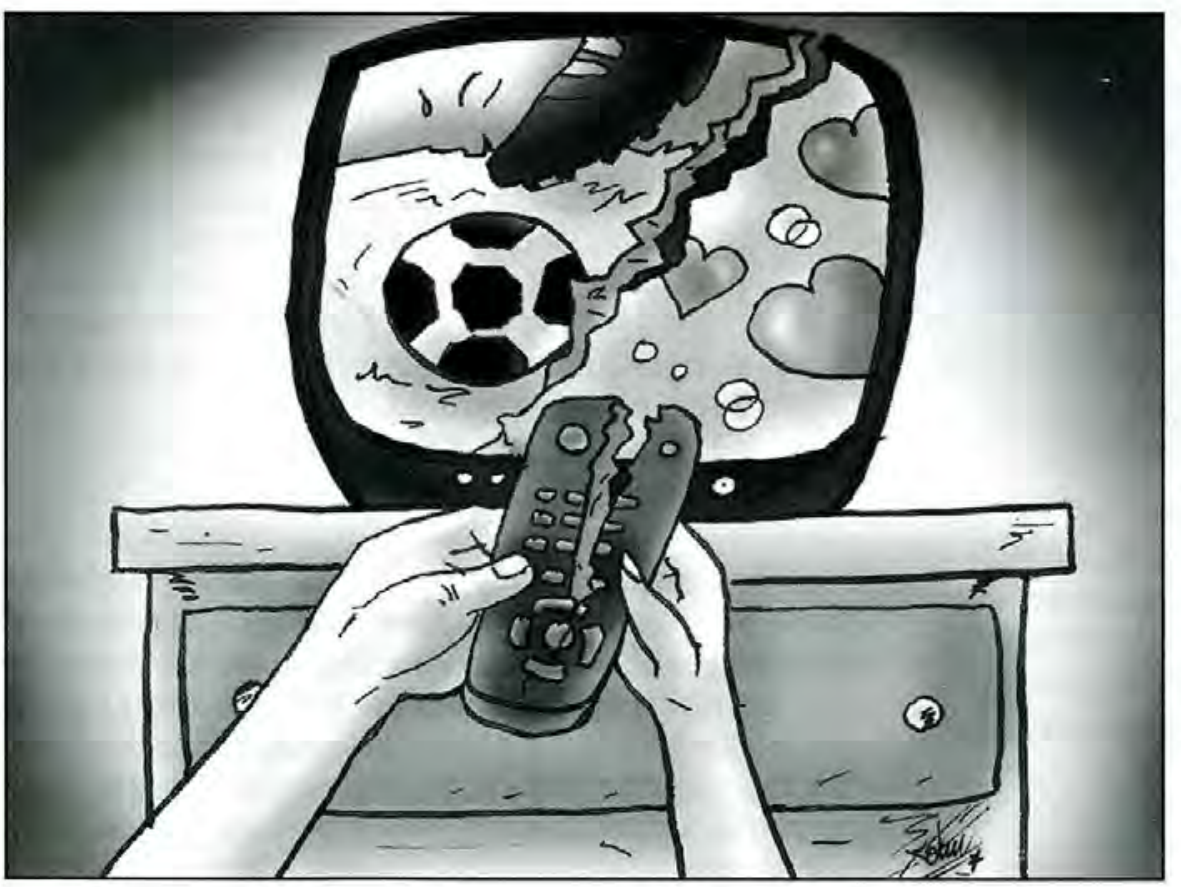

Tanto los hombres como las mujeres experimentan el consumo como un proceso de formación y expresión de su identidad (Zukin y Smith, 2004). Sin embargo, como ya se mencionó, las investigaciones siguen reduciendo el análisis de la relación entre el consumo y el género a comparaciones en función del sexo. En estos estudios no se ha abordado el papel que juega la identidad de género en los procesos de apropiación y uso de productos culturales ofrecidos por los medios de comunicación.

Uno de los medios de comunicación que promueve en mayor medida el consumo, es la televisión. Tal como lo señala un informe presentado en el Mercado Internacional de Programas de televisión (MIPTV) en Cannes, Francia (Revista Latinoamericana de Comunicación Chasqui, 2006) el consumo de la televisión continúa en aumento. En dicho informe se menciona que el promedio de consumo mundial ascendió a tres horas y siete minutos por día, promedio similar a la duración media encontrada en Latinoamérica (tres horas y 16 minutos) siendo el máximo de más de cinco horas en Japón, seguido por Estados Unidos.

En Colombia, según la Encuesta de Calidad de Vida del Departamento Administrativo Nacional de Estadística de Colombia (2003), el televisor es el artículo que compran primero las familias después de la nevera. Aún los estratos más bajos incluyen el pago de televisión por suscripción en su presupuesto mensual. 
Para el presente estudio se escogió como objeto de análisis la televisión, teniendo en cuenta que sigue siendo el medio de comunicación de mayor consumo en el mundo, de más fácil acceso y, junto con la Internet, de gran poder comunicativo, en tanto que implica la utilización de varios canales sensoriales por parte del espectador. A continuación se exponen de manera resumida los indicadores de consumo cultural que se consideraron en la investigación.

\section{Expresiones de los consumos culturales}

Para los propósitos de esta investigaclón se tuvieron en cuenta tres expresiones del consumo televisivo: a) La Selección de los consumos culturales, definida como la elección que realiza la persona del programa televisivo de su preferencia; b) La Interpretación de los consumos culturales. Definida como la identificación y valoración que hacen las personas, de los contenidos de género que se presentan en los programas que consumen. Valoración en términos de la existencia de comportamientos equitativos o sexistas y c) La Producción de consumos culturales, entendida como la creación o invención de fragmentos o totalidades de productos culturales que se podrian ofrecer para el consumo público por televisión.

\section{¿Qué sabemos sobre selección, interpretación y producción de consumos en términos de género?}

Usualmente se menciona que las mujeres tienden a consumir preferentemente novelas y dramafizados, mientras los hombres ven con mayor frecuencia programas deportivos y noticieros. Aunque algunos estudios han encontrado diferencias en la selección que hacen hombres y mujeres de los programas

\section{Aprendizajes}

televisivos, otros no han encontrado lo que comúnmente se considera preferencias femeninas, y en cambio encuentran que variables comola clase social yla generación se constituyen en importantes moderadores de qué y cómo ven las mujeres televisión (Yarto y Lozano, 2002).

En cuanto a la interpretación, los estudios generalmente la examinan en la tele-audiencia femenina. Por ejemplo, Muramatsu (2002) encontró que en la década de los 70 las mujeres japonesas se identificaban con algunos de los valores comunes descritos en los dramas de televisión, mientras que en los 90 surgió una visión más crítica de las audiencias femeninas acerca de los contenidos mediáticos. En un estudio sobre el discurso de la telenovela realizado en Colombia con 2400 estudiantes universitarios de ambos sexos (Martinez, Muñoz y Asqueta, 2006), se encontró que la mayoria interpretaron este género de ficción, como reproductor de modelos tradicionales pero, al mismo tiempo, como excelente pasatiempo. Este hecho evidencia una ambivalencia entre el reconocimiento cognifivo de lo que promueve y la seducción que les produce este tipo de programas.

En el caso de los estudios sobre los productos que los medios de comunicación ponen a disposición del público y su relación con el género, llevados a cabo en los años sesenta y principios de los setenta (Mattelart, 1977: Santa Cruz y Erazo, 1980), se encontró que los medios impresos (fotonovelas, novelas de amor y las llamadas revistas femeninas) proponian una identidad femenina caracterizada por la subordinación, los roles domésticos y privados, donde el prototipo físlco podria describirse como una mujer blanca, de buena posición social, alta, rubia, esbelta, en un claro contraste con el modelo real de la mujer latinoamericana de los sectores populares (Charles, 1996). 
Aunque hoy la situación ha cambiado en algunos aspectos, el último informe realizado en España (López, 2005), sobre las representaciones de género que transmiten los productos noticiosos de televisión, encontró que las mujeres suelen ser representadas como victimas, sin profesión declarada y en muchas ocasiones sin nombre y apellido. En contraste, los hombres son representados generalmente como agresores, siendo los deportistas y los políticos los grupos de hombres que se mencionan con mayor frecuencia en la televisión española. Igualmente, el Proyecto de Monitoreo Global de los Medios realizado el 16 de febrero de 2005 (WACC, 2005) encontró que las mujeres sólo constituyen el $21 \%$ de las personas que figuran en las noticias en el mundo y sólo el 10\% de estas noticias tienen a las mujeres como el foco central. El $86 \%$ de las personas que aparecen como expertas son hombres, las noticias son reporteadas y presentadas principalmente por hombres, mientras que el $57 \%$ de las presentadoras de televisión son mujeres.

Co mo se puede deducir de lo anterior, se encuentra evidencia de marcadas diferencias en los productos televisivos cuando el análisis se centra en el sexo de las personas, especialmente como consumidoras, pero se carece de información directa sobre las relaciones entre las construcciones de género y el consumo.

El análisis de género en las personas como productoras es prácticamente inexistente o desconocido. A pesar de que existe información sobre cómo los consumos culturales reproducen ciertas identidades de género no se ha estudiado cómo se relacionan las identidades de género de quienes están a cargo de la creación de consumos culturales televisivos con sus producciones. Estudiar la influencia de las concepciones acerca de la identidad de género sobre la producción de contenidos mediáticos es importante con el fin de conocer los perfiles de género de quienes se expresan en las producciones mediáticas y proponer pautas de acción en la educación sobre género y medios dirigida a comunicadores y realizadores. En este sentido, vale la pena citar el comentario publicado en una revista de círculación nacional, de un conocido llbretista colombiano, Fernando Gaitán, quien ha escrito los libretos de varias telenovelas de gran éxito internacional como Betty la fea, Café y Hasta que la plata nos separe, en relación con su fuente de inspiración. Según el llbretista en esta última producción, su principal fuente de inspiración son la gran mayoria de las colombianas: "las colombianas son muy bravas, son maravillosas, sensuales, hogareñas, solidarias, excelentes esposas, pero son bravas, algo malo tenían que tener..." (Colsubsidio, Vivir Mejor, 2006, pág. 40). Características que coincidencialmente son las mismas que hacen parte del estereotipo femenino tradicional.

Si asumimos que la mayoria de las diferencias entre los sexos son construcciones culturales, reforzadas social y psicológicamente, es necesario revisar cuáles son las construcciones de género, Independientemente del sexo de las personas, que se relacionan con unos tipos de consumos particulares, con unas interpretaciones y unas producciones sexistas o equitativas en términos sexuales. De especial relevancia es el estudio de las producciones realizadas por los comunicadores sociales y los periodistas, quienes tienen importante influencia en la selección, interpretación y producción de los consumos que se transmiten a través de los medios de comunicación.

El objetivo del presente estudio es, por lo tanto, avanzar en el conocimiento sobre esta temática dando respuesta a las siguientes preguntas investigativas: ¿Cómo se relaciona la identidad de género con la selección. interpretación y producción de material mediático 

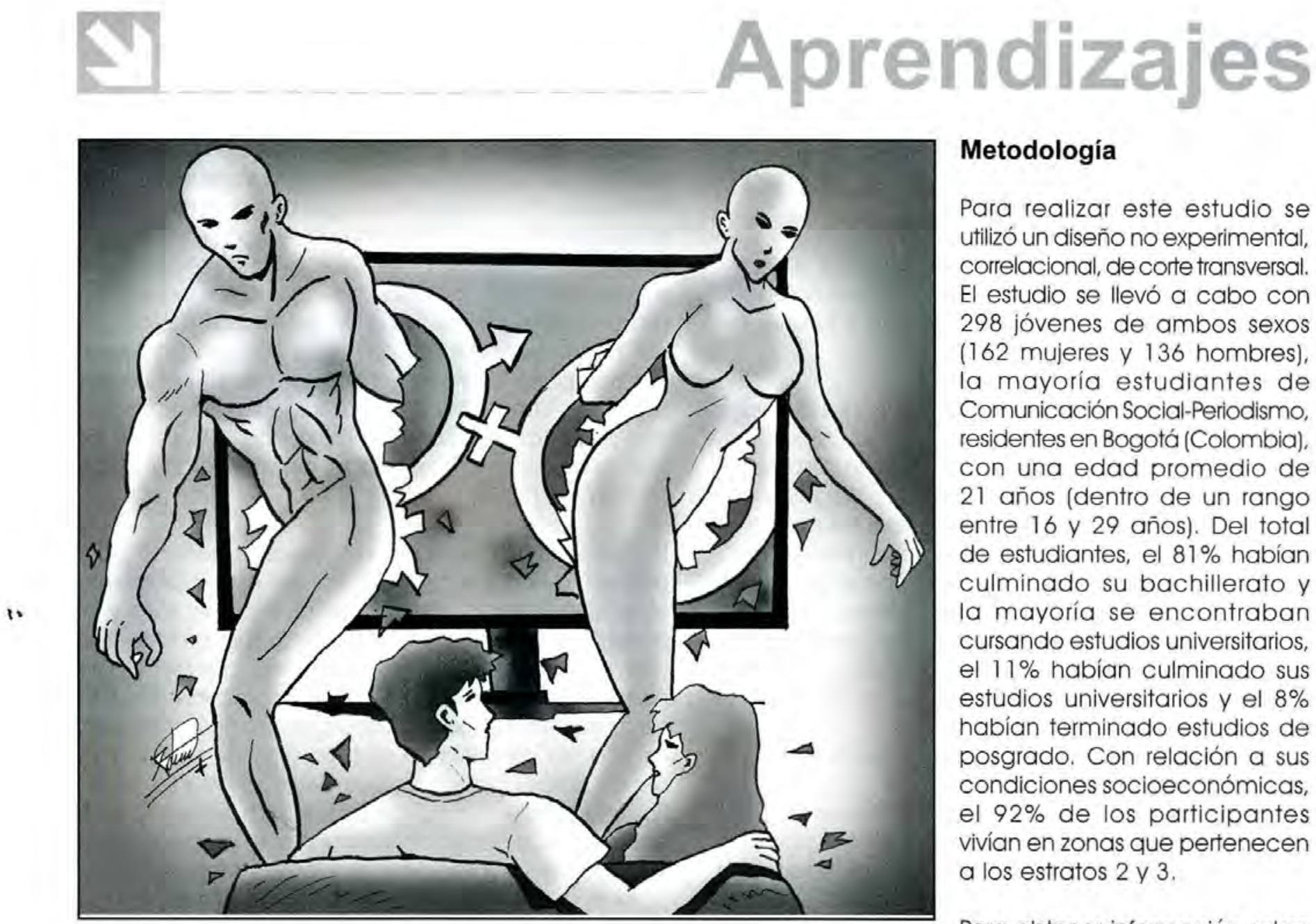

\section{Metodología}

Para realizar este estudio se utilizó un diseño no experimental, correlacional, de corte transversal. El estudio se llevó a cabo con 298 jóvenes de ambos sexos (162 mujeres y 136 hombres), la mayoría estudiantes de Comunicación Social-Periodismo, residentes en Bogotá (Colombia), con una edad promedio de 21 años (dentro de un rango entre 16 y 29 años). Del total de estudiantes, el $81 \%$ habian culminado su bachillerato y la mayoría se encontraban cursando estudios universitarios, el $11 \%$ habian culminado sus estudios universitarios y el $8 \%$ habian terminado estudios de posgrado. Con relación a sus condiciones socioeconómicas, el $92 \%$ de los participantes vivían en zonas que pertenecen a los estratos 2 y 3 .

Para obtener información sobre las variables del estudio se utilizó

puesto a disposición de las audiencias como consumo cultural televisivo?¿Puede la identidad de género ser un factor más influyente que la edad y el nivel educativo en la elección, interpretación y producción que las personas realizan del material mediático puesto a su disposición como consumo cultural televisivo? un cuestionario de auto-informe que incluía un total de 263 reactivos. El cuestionario constaba de cuatro cuadernillos los cuales incluyeron preguntas sobre las variables sociodemográficas (edad, sexo, nivel educativo), las variables predictoras (identidad con el sexo, identidad con el género, roles de género y actitudes sexistas) y las variables criterio (selección, interpretación y producción de consumos televisivos). Con el fin de controlar el sesgo de respuesta se 
elaboraron tres versiones del instrumento modificando el orden de presentación de los seis cuadernillos que contenian las escalas de medida.

El cuadernillo 1 incluía 17 preguntas sobre las variables sociodemográflcas y sobre la selección de los consumos televisivos preferidos, horarios, contenidos y razones de preferencia.

El cuadernillo 2 constaba de 12 afirmaciones definidas para medir la identidad con el sexo y la identidad con el gènero, a partir de una adaptación de un instrumento inicialmente desarrollado por Cortés y Vargas Trujillo (2004). Un ejemplo de ítem sobre identidad con el sexo de esta escala es: "Me gusta ser mujer/hombre", Un ejemplo de ítem sobre identidad con el género es:

1. "Me disgusta tener que hacer cosas que se consideren tareas de mujer/hombre". Puntajes altos correspondian a la percepción de mayor compatibilidad o afinidad con el sexo o con la propia categoria de género.

El cuadernillo 3 incluia escalas para medir actitudes sexistas y roles de género. En total constaba de 43 afirmaciones, Dos ejemplos de ítems son: Las mujeres necesitan mayor cuidado y protección que los hombres, El hombre es quien debe defender a la familia. Puntajes altos en la escala de actitudes sexistas indicaban una mayor disposición de la persona a tratar de manera desigual a las personas por el hecho de ser biológicamente hombres o mujeres. Mientras que puntajes altos en la escala de roles de género correspondian al mayor grado de conformidad con las normas y expectativas sociales acerca de cómo deben ser y comportarse las personas en función de su sexo.

El cuademillo 4 constaba de una escala diseñada para evaluar la interpretación de las relaciones de género que según el participante se presentaban o no en el programa escogido.
Incluia 31 afimaciones. Un ejemplo sobre comportamiento equitativo es: El programa refuerza la igualdad entre los sexos, Un mayor puntaje indicaba mayor grado de acuerdo con que el comportamiento se presentaba en el programa elegido. Los programas que se propusieron para la interpretación se seleccionaron teniendo en cuenta los índices de mayor audiencia en el mes en que se realizó la aplicación del instrumento. Los programas seleccionados fueron por una parte las telenovelas: Hasta que la plata nos separe con un $12.4 \%$ de audiencia y Los Reyes con un $11.5 \%$, por otra el reality Factor $X s$ con 10\% según los índices de audiencia reportados por www. TotalTV, Ibope - Colombia (2006).

El Cuadernillo 5 incluía el instrumento diseñado para evaluar la variable producción, en este instrumento se solicitó a cada participante definir a) el sexo y b) el perfil protagónico de una proxima producción televisiva. Una vez definido el sexo del personaje protagónico el participante debía marcar de una lista de atributos aquellos que prefería que lo caracterizaran. La lista de atributos se obtuvo a partir del análisis de los datos cualitativos que arrojó el estudio piloto. Posteriormente, dichas características se agruparon en categorías, teniendo en cuenta el procedimiento utilizado por Ludlom y Mahalik (2002) y Mahalik (2005), para la construcción de las escalas de medición de conformidad con normas femeninas y masculinas.

La versión final del instrumento, es un conjunto de 126 atributos agrupados en 21 categorías: agresividad, sociabilidad, alto control emocional, bajo control emocional, orientación de logro, no-orientación de logro, culdado de otros, búsqueda del rlesgo, prevención del riesgo, autovaloración, autodevaluación, fortaleza fisica. debilidad física, apariencia física deseable, actividad sexual, pasividad sexual, buen humor, comportamiento no ético y orientación sexual. Los participantes conocian 
Tabla 1. Resultados del análisis de confiabilidad de las escalas de medida

\begin{tabular}{|l|c|c|c|c|c|}
\hline \multicolumn{1}{|c|}{ Escala } & $\begin{array}{c}\text { Total ítems } \\
\text { inicial }\end{array}$ & $\begin{array}{c}\text { ítems } \\
\text { eliminados }\end{array}$ & $\begin{array}{c}\text { Total items } \\
\text { invertidos }\end{array}$ & $\begin{array}{c}\text { Total ítems } \\
\text { final }\end{array}$ & Alfa \\
\hline $\begin{array}{l}\text { Identidad de } \\
\text { género }\end{array}$ & 3 & 0 & 1 & 3 &, 559 \\
$\begin{array}{l}\text { Auto concepto } \\
\text { sexual }\end{array}$ & 9 & 0 & 9 & 9 &, 692 \\
$\begin{array}{l}\text { Auto concepto de } \\
\text { género }\end{array}$ & 32 & 0 & 0 & 32 &, 930 \\
$\begin{array}{l}\text { Actitudes sexistas } \\
\text { Roles de género } \\
\text { Interpretación del } \\
\text { consumo cultural }\end{array}$ & 43 & 0 & 0 & 43 &, 965 \\
$\begin{array}{l}\text { Comportamientos } \\
\text { equitativos }\end{array}$ & 25 & 0 & 0 & 25 &, 903 \\
$\begin{array}{l}\text { Comportamientos } \\
\text { sexistas }\end{array}$ & 6 & 0 & 0 & 6 &, 743 \\
\hline
\end{tabular}

los atributos mas no las categorías que los agrupaban El puntaje para cada categoría corresponde a la suma de atributos marcados por el participante como caracteristicos de su personaje.

Cabe señalar que antes de llevar a cabo los análisis requeridos se evaluó estadísticamente, la capacidad de las escalas para medir de forma consistente y precisa la variable evaluada. Se encontró que el grado de homogeneidad entre los elementos de las escalas fue alto para la mayoría de ellas. En la tabla 1 se observa el alfa de Cronbach para cada escala.

\section{Resultados: análisis descriptivo Identidad de género}

En la tabla 2 se presentan los descriptivos para los indicadores de la identidad de género. Llama la atención que mientras la puntuación promedio más alta corresponde a la identidad con el sexo: 5,7, la identidad con el género sólo llega a 3,5. Aunque los jóvenes perciben un alto grado de compatibilidad con su sexo biológico (ser hombres o mujeres) tienden a percibir niveles intermedios de afinidad con su género (con lo que se espera socialmente que sean y hagan por ser hombres o mujeres). También es interesante observar, que las medias de las actitudes sexistas y de los roles de género tienden a ser bajas, Estos resultados indican, por una parte, una actitud poco favorable de los jóvenes participantes, a tratar de manera desigual a las personas por el hecho de ser biológicamente hombres o mujeres, por otra, un bajo grado de conformidad con las normas y expectativas sociales acerca de cómo deben ser y comportarse las personas en función de su sexo².

\section{Consumos culturales}

El análisis descriptivo para la selección de consumos televisivos muestra que los noticieros y los programas investigativos son el tipo de programa preferido por los participantes $(24.2 \%)$, lo cual seguramente está relacionado con el hecho de que la mayoria son estudiantes de cameras universitarias relacionadas con la Comunicación Social

${ }^{2}$ Sin embargo, cuando el análisis se realiza por sexo, los resultados del estudio, evidencian que los hombres presentan mayores niveles de conformidad tanto con actitudes sexistas como con roles de género, en comparación con las mujeres. 
Tabla 2. Estadísticos descriptivos para los indicadores de la identidad de género

\begin{tabular}{|l|c|c|c|c|c|}
\hline & N & Mínimo & Máximo & Media & DE \\
\hline $\begin{array}{l}\text { Identidad con el } \\
\text { sexo }\end{array}$ & 298 & 2.00 & 6.00 & 5,7036 &, 64248 \\
$\begin{array}{l}\text { Identidad con el } \\
\text { género }\end{array}$ & 298 & 1,56 & 6,00 & 3,5776 &, 88125 \\
$\begin{array}{l}\text { Actitudes } \\
\text { sexistas } \\
\begin{array}{l}\text { Roles de } \\
\text { género }\end{array}\end{array}$ & 298 & 1,00 & 5,19 & 2,5519 &, 88606 \\
\hline
\end{tabular}

y el Periodismo, Le siguen en orden de preferencia los seriados y películas (17.8\%), los dramatizados y novelas $(16.1 \%)$, los dibujos animados (14.8), los musicales con $8.7 \%$, los realities y deportes, cada uno con $5.7 \%$. Sólo $3 \%$ de los participantes mencionó que veía programas "educativos. Entre los programas que mencionaron con mayor frecuencia además de los noticieros, sobresalen: Los Simpsons, el seriado Sin tetas no hay paraíso y las novelas Hasta que la plata nos separe y Los Reyes. El horario en el que prefieren ver televisión es la noche (64\%).

En cuanto a la interpretación de consumos televisivos, tal como se observa en la tabla 3 , en promedio los jóvenes identifican tanto un índice moderado de comportamientos equitativos como de comportamientos no equitativos en los consumos propuestos.

\section{Tabla 3. Estadísticos descriptivos para los indicadores de} interpretación del consumo

\begin{tabular}{|l|c|c|c|c|c|}
\hline Indicador & $\mathbf{N}$ & Minimo & Máximo & Media & DE \\
\hline $\begin{array}{l}\text { Comp. } \\
\begin{array}{l}\text { Equitativos } \\
\text { Comp. }\end{array}\end{array}$ & 296 & 1 & 6 & 3,7688 & .93264 \\
Sexistas & 296 & 1 & 6 & 3.5830 & 1.08510 \\
\hline
\end{tabular}

Enloreferentealaproducción de consumos televisivos se encontró que el $60 \%$ de los participantes eligieron un personaje femenino para protagonizar su guion, Los datos indican, como se puede apreciar en la tabla 4 que los atributos que eligieron con mayor frecuencia para caracterizar al protagonista de su producción son: sociabilidad, apariencia física deseable, orientación al logro y buen humor.

\section{Identidad de género y selección de consumos}

Con el fin de examinar si los indicadores de identidad de género y el tema elegido como consumo cultural televisivo difieren en función del sexo de los participantes, se realizó un análisis de varianza de dos factores.

En este análisis se encontraron diferencias significativas en las medias de las actitudes sexistas en función del tema elegido $F(7,267)=2,727, p=0,009^{3}$. Los

${ }^{3}$ En este anălisis también se encontraron diferencias significativas atribuibles al sexo en las medias de identidad con el género, $F$ $(1,267)=23,452, p=0,00$; las actitudes sexistas, $F(1,267)=$ 23,425, $\mathrm{p}=0.00$ y los roles de género $F(1,267)=14,338, p=$ 0.00 . Los hombres presentan puntajes significativamente más altos tanto en actitudes sexistas como en conformidad con roles de género comparados con las mujeres, mientras que ellas están significativamente menos conformes con su identidad de género comparadas con los hombres. 
jóvenes que escogen como primera opción programas informativos, independientemente de su sexo, son también quienes tienden a tener los más bajos puntajes en actitudes sexistas. Mientras que, tanto las mujeres que escogen como primera opción novelas, dramatizados y realities, como los hombres que escogen programas deportivos como primera opción, son quienes muestran mayores actitudes sexistas y mayor conformidad con los roles de género.

Las diferencias significativas observadas en los distintos indicadores de la identidad atribuibles al tema de preferencia son simllares tanto para hombres como para mujeres, es decir, no se encontró un efecto de interacción entre el sexo y el tema elegido.

Llama la atención que sólo las mujeres que eligieron programas deportivos son quienes expresan la menor conformidad en la escala de identidad con el género y con los roles de género, es decir, con lo que se espera social y tradicionalmente de hombres y mujeres. Esto es, las mujeres que se perciben menos conformes y satisfechas con las normas y expectativas de género prefieren programas televisivos que, de acuerdo con estudios previos, usualmente eligen las audiencias masculinas.

\section{¿Qué tanto explica la identidad de género, comparada con la edad y el nivel educativo la interpretación de los consumos culturales televisivos?}

Para responder a este interrogante se realizaron análisis de regresión por pasos sucesivos para todo el grupo y separadamente por sexos. Se encontró que la percepción que los jóvenes tienen con relación a las actitudes sexistas y los roles de género, así como su edad sí explican, aunque en una mínima parte la interpretación que hacen de los consumos, independientemente que sean hombres o mujeres. El factor actitudes sexistas
Tabla 4. Atributos asignados al perfil del protagonista del guion

\begin{tabular}{|c|c|c|c|c|c|}
\hline $\begin{array}{l}\text { Caracteristicas } \\
\text { del protagonista }\end{array}$ & $\mathbf{N}$ & Mínimo & Máximo & Media & $\mathrm{DE}$ \\
\hline $\begin{array}{l}\text { Agresividad } \\
\text { Sociabilidad }\end{array}$ & $\begin{array}{l}298 \\
298\end{array}$ & $\begin{array}{l}00 \\
00\end{array}$ & $\begin{array}{c}8 \\
14\end{array}$ & $\begin{array}{c}.7013 \\
5.7148\end{array}$ & $\begin{array}{l}1.17840 \\
4.25087\end{array}$ \\
\hline $\begin{array}{l}\text { Alto control } \\
\text { emocional }\end{array}$ & 298 & 00 & 3 & .6477 & .74310 \\
\hline $\begin{array}{l}\text { Bajo control } \\
\text { emocional }\end{array}$ & 298 & 00 & 13 & 1.7248 & 1.98601 \\
\hline $\begin{array}{l}\text { Orientación al } \\
\text { logro }\end{array}$ & 298 & 00 & 14 & 5.8658 & 3.82295 \\
\hline $\begin{array}{l}\text { No-orientación al } \\
\text { logro }\end{array}$ & 298 & 00 & 7 & .6779 & 1.01350 \\
\hline Cuidado de otros & 298 & 00 & 8 & 1.9262 & 1.95089 \\
\hline $\begin{array}{l}\text { Búsqueda del } \\
\text { riesgo }\end{array}$ & 298 & 00 & 3 & .9832 & .97600 \\
\hline $\begin{array}{l}\text { Prevención del } \\
\text { riesgo }\end{array}$ & 298 & 00 & 4 & 1.2450 & 1.03981 \\
\hline Autovaloración & 298 & 00 & 6 & 1,8893 & 1.34258 \\
\hline Autodevaluación & 298 & 00 & 4 & .2919 & .62926 \\
\hline Fortaleza fisica & 298 & 00 & 3 & 1.0906 & $\begin{array}{l}1.05498 \\
\end{array}$ \\
\hline Debilidad física & 298 & 00 & 2 & 1309 & .35721 \\
\hline Físico deseable & 298 & 00 & 6 & 2.6007 & $\begin{array}{l}1.76525 \\
0888 ?\end{array}$ \\
\hline Actividad sexual & 298 & 00 & 4 & .8658 & 88882 \\
\hline Pasividad sexual & 298 & 00 & 4 & .5201 & .73941 \\
\hline Buen humor & 298 & 00 & 5 & 2.2517 & 1.48406 \\
\hline $\begin{array}{l}\text { Comportamiento } \\
\text { no ético }\end{array}$ & 298 & 00 & 3 & .2752 & .64468 \\
\hline $\begin{array}{l}\text { Heterosexual } \\
\text { Homosexual }\end{array}$ & $\begin{array}{l}298 \\
298\end{array}$ & $\begin{array}{l}00 \\
00\end{array}$ & $\begin{array}{l}1 \\
1\end{array}$ & $\begin{array}{l}.3022 \\
.0638\end{array}$ & $\begin{array}{l}.45990 \\
.24473\end{array}$ \\
\hline
\end{tabular}


y roles de género tiene un peso significativo qunque minimo en la identificación de comportamientos equitativos en los programas, mientras que la edad lo tiene en la identificación de comportamientos sexistas de los programas seleccionados.

Llama la atención que el nivel educativo, es decir, el hecho de ser bachiller, estudiante universitario o estudiante de posgrado, no se encuentra como variable explicativa en la identificación de comportamientos equitativos o sexistas en la interpretación de los consumos hechos.

En cuanto a la producción, se encontró que la alta identidad con el sexo, es decir, la percepción de mayor afinidad y satisfacción con el hecho de ser hombre - mujer es una variable que explica la atribución al personaje protagónico de las caracteristicas de orientación al logro e interés por cuidar a otros 4 .

Un hallazgo interesante es que el estar de acuerdo con acfitudes sexistas y roles de género tradicionales explica la atribución al personaje protagónico de caracteristicas no éticas. En los hombres adicionalmente, explica la atribución de agresividad y no prevención de riesgos, mientras que en las mujeres la atribución de características relacionadas como un fisico deseable, pasividad sexual y auto-devaluación.

\footnotetext{
"Se encontraron en este punto algunas diferencias por sexo. En los hombres una alta conformidad con su sexo también hace parte del modelo que explica la atribución al personaje protagónico de las siguientes caracteristicas: autovaloración e interés por cuidar de otros, mientras que en las mujeres una alta conformidad con su sexo también explica en parte la atribución de caracteristicas como sociabilidad, prevención del riesgo, fortaleza fisica y buen humor. Este y otros resultados diferenciales merece un análisis más detallado en otra oportunidad.
}

Llama la atención que únicamente en los hombres, la edad se encuentra como parte del modelo explicativo de la atribución de características como la autodevaluación y la agresividad atribuida al personaje principal. Tampoco aqui el nivel educativo explica lo relacionado con la producción.

\section{Conclusiones}

Los resultados presentados en este texto, evidencian la complejidad del estudio de la identidad de género cuando se quiere trascender los supuestos teóricos para pasar a determinar empíricamente las relaciones entre el género y el consumo. Tal como lo señala Udry (2001), la identidad con el gènero involucra múltiples factores que interactúan entre si, algunos biológicos, otros culturales y otros individuales, sin embargo las limitaciones de los diseños de investigación hacen imposible considerar todas las variables simultáneamente ${ }^{5}$.

Los hallazgos revelan que sí existen relaciones significativas entre los indicadores de la identidad de género examinados en este estudio y la selección, interpretación y producción de consumo cultural televisivo, Por lo tanto, algunas de las diferencias que las investigaciones han encontrado y en algunas ocasiones atribuido al sexo de las personas realmente no tienen que ver con el sexo por sí mismo, sino con las construcciones de genero que las personas han elaborado y asimilado en sus historias particulares y en los contextos culturales en los que se desenvuelven.

El ser ubicado o ubicarse en una categoría sexual particular, es un elemento importante pero no determinante para explicar la selección, interprefación

${ }^{5}$ El estudio completo incluye algunas consideraciones teóricas en torno a la definición de género. 


\section{Y Aprendizajes}

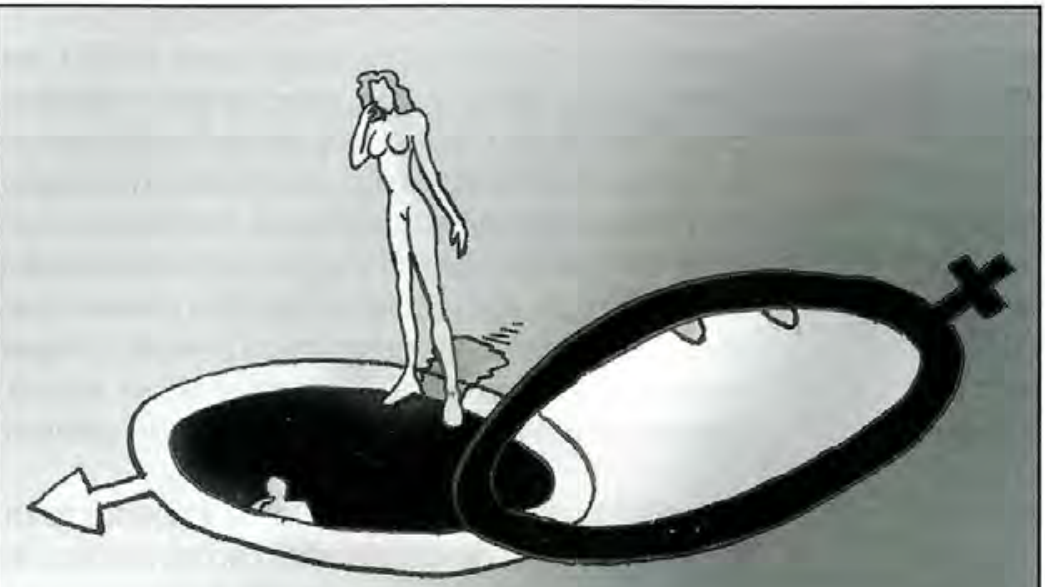

1. Es preciso que los estudios sobre género y comunicación deslinden el sexo de las construcciones de género con el ánimo de aportar a una comprensión mayor de los fenómenos estudiados y no seguir atribuyendo tendencias por sexo como si todos los hombres y todas las mujeres, independientemente de sus construcciones de género, se pudieran homogeneizar en categorías separadas.

2. El estudio plantea la necesidad de persistir en la tarea de precisar conceptual y operacionalmente el constructo identidad de género. Los datos muestran la urgencia de que los investigadores que trabajan el tema del géneroy, particularmente de la identidad de género expliciten los supuestos teóricos y empíricos que fundamentan sus técnicas de recolección de información. Evidentemente, las conclusiones de los estudios difieren en función del indicador que usemos de la identidad de género. Sólo en la medida que compartamos los supuestos subyacentes a los constructos que examinamos y las medidas que usamos podemos avanzar en el desarrollo del conocimiento.

y producción de consumos en términos de género. De hechø, el considerar únicamente el sexo en los estudios sobre género es insuficiente y en ocasiones puede confundir más que aclarar las relaciones entre las construcciones de género y el consumo.

Los hallazgos del presente estudio que se sintetizan en este artículo permiten concluir que:

3. En cuanto a las relaciones entre género y selección de consumos, las diferencias que generalmente se establecen entre el consumo de novelas y dramatizados por parte de las mujeres y el consumo de programas deportivos y series de televisión por parte de los hombres, se presentan según el presente estudio, tanto en mujeres como en hombres que muestran altos puntajes en actitudes sexistas. Los resultados de los estudios que han analizado los contenidos de telenovelas 
y programas deportivos, evidencian que dichos contenidos son altamente estereotipados y sexistas (Vargas, E; Barrera, F; Burgos, C y Daza, B (2004). En esta investigación se encontró que son los jóvenes que informan una mayor disposición a tratar de manera desigual a las personas por el hecho de ser biológicamente hombres o mujeres, quienes escogen también consumos fuertemente estereotipados sexualmente. Mientras que llama la atención que hombres y mujeres que reportan alta afinidad con su sexo y bajas actítudes sexistas, escogen como primera opción programas noticiosos e investigativos, los cuales aunque no están exentos de contenidos sexistas no suelen ser tan abiertamente estereotipados. Adicionalmente, el hecho de que las mujeres con bajas actitudes sexistas y quienes desaprueban los roles sexuales tradicionales prefieran como primera opción programas deportivos, los que usualmente se han asociado con preferencias masculinas, puede indicar el deseo de acceder aunque sea simbólicamente, tal como lo plantea Larrain (2001), a un grupo imaginado. Estos resultados muestran que las diferencias encontradas en otros estudios en el consumo televisivo en función del sexo, pueden ocultar las razones, que por lo menos en términos de género, contribuyen a entender la selección de dichos consumos.

4. Con rélación a la interpretación, los resultados permiten concluir, que el estar de acuerdo o no con las actitudes sexistas y los roles tradicionales de género son variables predictoras significativas, aunque pequeñas en ambos sexos, de identificación y valoración que hacen los jóvenes de los comportamientos equitativos y sexistas en los consumos televisivos interpretados. Si se tiene en cuenta que gran parte de los mensajes que emiten los medios masivos de comunicación son abiertamente sexistas (Herold y Foster, 1975; Luke, 1996: López, 2001), y que como se encontró en el presente estudio, aquellos jóvenes que están de acuerdo con el trato desigual en función del sexo, no interpretan como sexistas los contenidos que se emiten en los consumos que ven, el limitarse a Informar o divulgar sobre el sexismo presente en los contenidos de los programas puede no tener mayor efecto en estos jóvenes, si no se trabaja directamente sobre sus cogniciones de género.

5. La edad también es una variable importante en la identificación de comportamientos sexistas de los consumos televisivos por parte de los hombres. Los más jóvenes expresan puntuaciones más altas en actitudes sexistas pero menor identificación de sexismo en los contenidos vistos, las razones pueden ir desde la falta de experiencia e información suficiente sobre el tema, hasta la existencia de relaciones estrechas entre dichas actitudes sexistas y su visión de la masculinidad. lo cual les impide percibir e interpretar como sexistas estos comportamientos presentes en los contenidos de los consumos preferidos. Se observa que a medida que aumenta la edad de los jóvenes éstos muestran mayor sensibilidad para identificar comportamientos sexistas en los consumos televisivos. Estos resultados son congruentes con otros estudios (Cournoyer y Mahalik, 1995), y con los planteamientos que afirman una mayor flexibilidad conceptual de los jóvenes para captar diferentes dimensiones y perspectivas de la realidad lo que a su vez favorece el reconocimiento y cuestionamiento de las normas, los roles y las expectativas de género (Fernández, 1996). 
6. Preocupa encontrar que el nivel educativo (educación secundaria, pregrado o posgrado) no está asociado con la interpretación en términos de género de dichos consumos; una posible expllcación puede tener que ver con el hecho de que ni los programas de secundaria, ni los programas de Comunicación Social-Periodismo promueven competencias para la recepción critica de consumos culturales con perspectiva de género. Aunque existen investigaciones diversas sobre el tema en varias facultades, realmente falta mucho para que los planes de estudio, los proyectos curriculares y los programas de cada curso incluyan la perspectiva de género. La mayoría de las veces el interés sobre el tema se reduce a un sector de docentes

7. En cuanto a la producción, el tipo de identidad de género se traduce en características diferenciales asignadas al personaje principal. La investigación permite concluir que tanto los hombres como las mujeres que presentan una mayor satisfacción con su sexo asignan significativamente mayor número de características al protagonista relacionadas con la orientación al logro e interés por cuidar a otros. Se podría concluir que tanto en hombres como en mujeres, estar conforme con el propio sexo puede ser parte de la satisfacción consigo mismo en general y por lo tanto esto se proyecta en un mayor uso de atributos deseables socialmente. Mientras que hombres y mujeres que presentan mayores

\section{Aprendizajes}

actitudes sexistas y conformidad con roles de género tienden a atribuir mayor número de características de comportamiento no ético al personaje protagónico creado 6 . Estar conforme con un trato desigual de las personas en función del sexo y con los roles de género tradicionales, juega un papel en la producción de atributos no deseables socialmente, como la autodevaluación y el comportamiento no ético.

8. Sise tiene en cuenta que la investigación se realizó preferentemente con estudiantes, profesionales, comunicadores sociales y periodistas, los cuales deben estar en capacidad de brindar y producir información y contenidos con la mayor objetividad y ética posible, sin discriminación alguna, en este caso por sexo, preocupa el hecho de encontrar que estos jóvenes aún presentan acuerdo con diversas actitudes sexistas y roles de género tradicionales, no identifican fácilmente contenidos sexistas en los consumos televisivos y la producción realizada se ve influida por construcciones de género inequitativas. Comunicadores y periodistas deben ser consciented de sus concepciones de género ya que como consumidores y como productores no actúan en abstracto sino que por el contrario sus acciones son muy concretas y reales, contribuyendo a construir significados y cultura.

También en este aspecto se encontraron diferencias entre hombres y mujeres que llaman la atención. Los hombres que presentan mayor nivel de actitudes sexistas y mayor conformidad con los roles de género tradicionales tienden a atribuir mayor número de caracteristicas agresivas y de no prevención de riesgos a su personaje, en el caso de las mujeres tienden a atribuir mayores caracteristicas de autodevaluación, físico deseable y pasividad sexual a su personaje. 
9. En estudios a futuro, que combinen metodologias tanto cuantitativas como cualitativas, se puede examinar si la producción de consumos culturales varía en función de los procesos de formación a los que son expuestas las personas. Por ejemplo. a traves de estudios cuasiexperimentales podria contrastarse la producción de personas que participan en procesos de formación con enfoque de género y los que no, lo que unido a otros factores podría transformar constructivamente los mensajes mediáticos y de esta forma ofrecer alternativas a futuro, sin dejar de lado la innegable influencia de lo indices de audiencia y los intereses económicos en la producción mediática (Bordieu, 1997).

10. Se perfilan algunos cambios en las cogniciones sobre género en los jóvenes, sin embargo, ahora el reto es identificar estrategias que permitan avanzar del discurso sobre equidad al de los factores contextuales que mantienen los comportamientos sexistas. Por ejemplo, a través de la inclusión planeada y consistente de prácticas y experiencias de relaciones equitativas en situaciones de la vida cotidiana.

11. Los resultados muestran también, que toda labor tendiente a reducir las actitudes sexistas puede convertirse en una importante contribución para mejorar la convivencia y la resolución pacífica de los conflictos, en la medida en que el trabajo sobre este aspecto puede al mismo tiempo disminuir la aceptación del uso de la agresión y la fuerza como caracteristicas deseables en las personas y particularmente en los personajes presentados a la teleaudiencia en los contenidos televisivos.
12. Con relación a la producción de consumos este estudio tambièn reafirma la necesidad de involucrar a productores, guionistas y realizadores de televisión en la producclón de consumos culfurales que muestren perfiles de personajes de hombres y mujeres con caracteristicas más pluralistas que las presentadas hasta el momento,

13. Una mención especial merece el hecho de queen esta muestra de jóvenes, la mayoria procedentes de niveles socio-económicos bajos (estratos 2 y 3), se observaron puntuaciones promedio de actitudes sexistas relativamente bajas. Otros estudios han encontrado que los miembros de estos grupos sociales tienden a informar niveles altos de actitudes sexistas. Este resultado se puede atribuir al hecho de que esta población, a pesar de sus limitaciones económicas, ha tenido acceso a la educación superior. Estudios posteriores deberán analizar si el acceso a la educación universitaria establece diferencias en las actitudes sexistas de los jóvenes de niveles socioeconómicos menos favorecidos.

14. Finalmente, se sugiere que la Política de Género de los goblernos locales, municipales y regionales involucre en el diseño de sus programas, estrategias acordes con la población joven y tenga en cuenta las particularidades en la selección, interpretación y producción de consumos culturales que hacen los jóvenes desde la perspectiva de género. 


\section{Aprendizajes}

\section{Limitaciones del estudio}

Este estudio aporta información relevante sobre la identidad de género y los consumos culturales, no obstante, es pertinente señalar algunas de sus limitaciones. La primera de ellas se deriva de la complejidad que implica el estudio de la identidad, en general, y concretamente de la identidad de género. Responder a las preguntas del estudio exigió realizar los análisis separadamente por sexo. Es probable que en este esfuerzo hayan quedado sin examinar algunos datos o que algunos resultados no hayan sido suficientemente descritos en este informe, estos pueden dar lugar a nuevas consideraciones en un futuro próximo.

Una limitación adicional es la que se deriva de la falta de instrumentos válidos y confiables para medir la identidad de género. Si bien en este estudio, a diferencia de otras investigaciones, se procuró explicitar la definición conceptual y operacional de las variables definidas como indicadores de este constructo, probablemente las medidas adolecen de limitaciones que conviene tener en consideración al momento de examinar los resultados y de desarrollar estudios con objetivos similares.

Otra limitación se refiere a las características de la muestra. Si tenemos en cuenta que la identidad de généro requiere un análisis y una interpretación contextual, es importante realizar estudios con grupos de población diferentes al que participó en esta investigación. Además, dado que la mayoría de los jóvenes participantes estudian Comunicación Social es necesario explorar las relaciones entre la identidad de género y el consumo en jóvenes con otras condiciones sociales y educativas. $[$ ?

\section{Bibliografia}

BONDER, G., "Las mujeres ante los medios: ampllando las opciones", en Mujer y comunicación. Una Alianza posible, Buenos Aires, WACC-CEM, 1995.

BOURDIEU, P., Sobre la televisión, Anagrama, Colección Argumentos, Barcelona, 1997.

BUSSEY, $K$, y BANDURA, A., Social Cognitive of gender Development and Differentiation, (4) 676-713, American psychological Association, 1999.

CAZÉS, D., La perspectiva de género. Guía para diseñar, poner en marcha, dar seguimiento y evaluar proyectos de investigación y acciones públicas y civiles, México. CONAPO - PRONAM, 1998.

COLSUBSIDIO, Revista de la Caja Colombiana de Subsidio Famillar Vivir Mejor, Quebecor World, Bogotá. 2006, pág. 40.

CONNELL, B., "Masculinidades, Violencia y Paz", en www. hombresporlaigualdad.com/default.htm, 2000.

CHARLES, M., "El espejo de venus: una mirada a la investigación sobre mujeres y medlos de comunicación", en Revista Signo y Pensamiento Núm. 28 (XV), Bogotá, Pontificia Universidad Javeriana, 1996, págs، 37-50.

, "El Género en la televisión", en Revista Signo y Pensamiento, Bogotá, Pontificia Universidad Javeriana, 2002.

CHASQUI Comunica, Org., "Sube el tiempo de permanencia ante el televisor", en Revista latinoamericana de comunicación, Núm, 94, 2006, Revisado en agosto 2 de 2006.

DANE, Departamento Administrativo Nacional de Estadística, Encuesta de Calidad de Vida, 2003. 
EXPÓSITO, MOYA y GLICK, "Sexismo ambivalente. Cognición y correlatos", en Revista de Psicología Social, núm. 13(2), 1998, págs. 159-169.

EGAN, S. y PERRY, D. " "Gender Identity A Multidimestional Analysis with Implications for Psychosocial Adjustment", en American Psychological Association, núm. 37 (4) 2001, págs، 451-463.

FERNÁNDEZ, J., Varones y Mujeres. Desarrollo de la doble realidad del sexo y del género, Madrid, Ed. Psicología Pirámide, 1996.

FLORES, S., y SÁNCHEZ, A., "Estereotipos demográficos y ocupacionales de la mujer y el hombre en la televisión mexicana", en XIII Anuario de Investigación de la

w. Comunicación CONEICC, Editora Maria Antonia Rebeil Corela, Instituto Tecnológico de Monterrey Campus Monterrey, 2006, págs. 257-271.

GARCÍA, N., El consumo cultural en América Latina, El consumo cultural. Una propuesta teórica, Bogotá, Convenio Andrés Bello, 1999, pág. 42.

LARRAIN, J., "El concepto de identidad", en Identidad chilena، Cap. 1. Ed. Lom Satiago de Chile, 2001, pag. 21-48.

LÓPEZ, P., Segundo informe sobre representaciones de género en los informativos de radio y televisión, Instituto oficial de radio y televisión, Madrid, 2005.

LOZANO, C. El género y el nivel socioeconómico como mediaciones en el consumo de noticleros televisivos en México, Zer, núm. 9, Universidad del país Vasco, 2001.

LUDLOW, L., \& MAHALIK, J., "Congruent Between continuum of Masculinity and the Rasch Model: Examining the Conformity to Masculine Norms Inventory", en Journal of Applied Measurement, 2002, págs. 205-221.

LUKE, C., Introduction Carmen Luke (ed), Feminism and Pedagogies of Everyday Life, New York, State University of New York press, 1996

MAHALIK, J., MORRAY, E., COONERLY, F., LUDLOW, L., SLATTERLY, S., \& SMILER, A., Development of Conformity to Femenine Norms Inventory, Sex Roles núm. 52, 2005 , págs. 317-335.

MARAMATSU, Y., "Gender Construction through interactions between the media and audience in Japan", en International Journal of Japanese Sociology, 11, 2002, págs. 72-87.

MARTíN BARBERO, J., "Consumo cultural", en Revista Mediaciones núm. 1, Bogotá, Corporación Universitaria Minuto de Dios, 2003.

MARTINEZZ, B.; MUÑOZ, C, y ASQUETA, M., Erase una vez... Análisis crítico de la telenovela. Colección Gramáticas Mediatizadas, Facultad de Ciencias de la Comunicación, Bogotá Corporación Universitaria Minuto de Dios, 2006.

MATTELART, M., La cultura de la opresión femenina. México, Era, serie popular, 1977.

MCMILLAN, J.H., "Enhacing collage students'critical thinking: A rewiew studies", en Reserch Higher Education. 26 (1), 1987, págs. 3-29.

MOYA, PAÉZ, GLICK, FERNÁNDEZ Y POESCHEL, Feminismo y masculinidad un estudio cultural, 1997.

OROzCO, G., "Hay que hacer algo, pero no somos los indicados", en Ámbltos de mediación y supertemas en la 
televidencia de las noticias, Comunicación y sociedad. 27, DECs Universidad de Guadalajara, 1996.

, "Mexican mediations in TV news reception". en Jensen, $K_{\text {. }}$ (coord.) News of the World: World Cultures Look at Television News, Londres, Routledge, 1998.

- Travesías y desafios de la investigación de la recepción en América Latina, Barcelona, Portal del INCAM, Universidad Autónoma de Barcelona, 2001.

SANTA CRUZ, A, y ERAZO, V., Compropolitan. El orden transnacional y su modelo femenino, México, ILET, Nueva Imagen, 1980.

SUNKEL, G., "El Consumo cultural en la investigación en comunicaciones en América Latina" en Revista Signo y Pensamiento 23 (45), Bogotá, Pontifica Universidad Javeriana, 2004, págs. 9-24.

SZASZ, I., "Masculine Identity and the Meanings of Sexuality: A Review of research in Mexico" en Reproductive Health Matters, Vol. 6, núm. 12, 1998.

UDRY, R., "Feminist Crtics Uncover Determinism, Postivism, and Antiquated Theory", en American Sociological Review; Aug, 66, 4 ABI/INFORM Global, 2001.

VARGAS, E; BARRERA, F; BURGOS, C., y DAZA, B., "Influencia de los programas televisivos con contenido sexual sobre el comportamiento de los adolescentes", en Documentos CESO, 82, Bogotá, Facultad de Ciencias Sociales Universidad de los Andes, 2004, págs. 8-53.

WACC, Proyecto de Monitoreo Global de los Medios, Plegable informativo ¿Quién figura en las noticias? London, 2005.
WITT, Gender, Culture and Representation, Oxford University Press, 1996.

WORTMAN, A., Pensar las clases medias, Buenos Aires, La Crujia, 2003.

W.W.TotalTV, Ibope Colombia, 2006.

YART, C., y LOZANO, C., "Televisión, rutinas y vida cotidiana en mujeres de México, Guadalajara y Monterrey presentado", en XIV Encuentro nacional AMIC Tendencias de la Investigación de la Comunicación: Hacia la construcción de un nuevo escenario. Tequisquiapan, Querétaro, mayo de 2002.

ZUKIN, S., y SMITH, J., "Consumers and Consumption", en Annual Review of Sociology, 30, 2004, págs. 97-173. 